\title{
Ethical implications of digital images for teaching and learning purposes: an integrative review
}

\author{
This article was published in the following Dove Press journal: \\ Journal of Multidisciplinary Healthcare \\ 10 June 2015 \\ Number of times this article has been viewed
}

\section{Rachel Kornhaber ${ }^{1-3}$ \\ Vasiliki Betihavas ${ }^{4}$ \\ Rodney J Baber ${ }^{5}$}

'School of Health Sciences, Faculty of Health, University of Tasmania, Rozelle, NSW, ${ }^{2}$ School of Nursing, The University of Adelaide, Adelaide, SA, ${ }^{3}$ Severe Burns Injury Unit, Royal North Shore Hospital, St Leonards, NSW, ${ }^{4}$ School of Nursing, University of Sydney, Sydney, NSW, ${ }^{5}$ Discipline of Obstetrics, Gynaecology and Neonatology, Sydney Medical School, University of Sydney, Sydney, NSW, Australia
Correspondence: Rachel Kornhaber School of Health Sciences, University of Tasmania, Locked Bag 5052, Alexandria, NSW 2015, Australia

Email rachel.kornhaber@utas.edu.au
Background: Digital photography has simplified the process of capturing and utilizing medical images. The process of taking high-quality digital photographs has been recognized as efficient, timely, and cost-effective. In particular, the evolution of smartphone and comparable technologies has become a vital component in teaching and learning of health care professionals. However, ethical standards in relation to digital photography for teaching and learning have not always been of the highest standard. The inappropriate utilization of digital images within the health care setting has the capacity to compromise patient confidentiality and increase the risk of litigation. Therefore, the aim of this review was to investigate the literature concerning the ethical implications for health professionals utilizing digital photography for teaching and learning.

Methods: A literature search was conducted utilizing five electronic databases, PubMed, Embase (Excerpta Medica Database), Cumulative Index to Nursing and Allied Health Literature, Educational Resources Information Center, and Scopus, limited to English language. Studies that endeavored to evaluate the ethical implications of digital photography for teaching and learning purposes in the health care setting were included.

Results: The search strategy identified 514 papers of which nine were retrieved for full review. Four papers were excluded based on the inclusion criteria, leaving five papers for final analysis. Three key themes were developed: knowledge deficit, consent and beyond, and standards driving scope of practice.

Conclusion: The assimilation of evidence in this review suggests that there is value for health professionals utilizing digital photography for teaching purposes in health education. However, there is limited understanding of the process of obtaining and storage and use of such mediums for teaching purposes. Disparity was also highlighted related to policy and guideline identification and development in clinical practice. Therefore, the implementation of policy to guide practice requires further research.

Keywords: digital photography, ethics, education, informed consent, practice guidelines, health professionals, photography, teaching materials, health care

\section{Introduction}

Digital photography has simplified the process of capturing and utilizing digital images for health care professionals. The process of taking high-quality digital photographs has been recognized as efficient, timely, and cost-effective. ${ }^{1-3}$ In particular, the evolution of smartphone and comparable technologies has enabled digital photography to become a vital component of teaching and learning in the health care setting. ${ }^{4,5}$ Consultation and documentation, clinical education, patient and family education, and publications are four key domains where digital images are frequently utilized within the clinical setting. ${ }^{6}$ This can be attributed to the minimal cost involved, user friendliness, and the 
ability to produce high-quality images that are easily stored, downloaded, and distributed via a multitude of mediums. ${ }^{2}$ However, ethical standards in relation to digital photography for teaching and learning have not always been of the highest standard. The inappropriate utilization of digital images within the health care setting has the capacity to compromise patient confidentiality and increase the risk of litigation. ${ }^{7}$ In particular, health care personnel need to consider the ethical implications of digital technology within the health care setting. ${ }^{8,9}$ The very simplicity of modern digital photography has resulted, in some cases, in a relaxation of the usually applied guidelines of informed consent. ${ }^{9}$ An unequal power balance could exist between health care professionals and patients who may feel coerced into consenting to digital photography. ${ }^{10}$ Taking photographs of at-risk and vulnerable populations requires greater ethical responsibility. ${ }^{11}$ Ensuring that the patient's identity and privacy is not sacrificed for the reason of ease and convenience is of prime importance. Furthermore, misuse of digital images obtained in clinical settings is becoming an area of concern ${ }^{10,12}$ and brings into question and highlights issues surrounding privacy and confidentiality. Consent, when obtained, is often verbal and may not include an explanation conveying photography is not only for treatment-related purposes, but also for education., ${ }^{9,10,13,14}$ Consequently, there is a heightened need for guidance in relation to the use of digital photography within the clinical setting where such technology plays an increasingly prominent role. ${ }^{15}$

The application of digital photography in the clinical setting for the purpose of teaching and learning is poorly reported in the health care literature, ${ }^{16}$ and the authors have been unable to identify a single review that investigates digital photography in teaching and learning of health professionals. Therefore, the aim of this integrative review was to investigate the current literature concerning the ethical implications of digital photography for teaching and learning purposes within the health care environment.

\section{Methods}

The framework guiding this integrative review is based on Whittemore and Knafl's ${ }^{17}$ five stages: problem identification, literature search, data evaluation, data analysis, and presentation. A systematic search was conducted using PubMed, Embase, Cumulative Index to Nursing and Allied Health Literature, Educational Resources Information Center, and Scopus. The references of all potential papers retrieved were examined to identify any additional papers fulfilling the inclusion criteria that may have been missed by the electronic searching strategy. Boolean connectors were used to combine search terms such as health care, medical, image*, smartphone*, digital, photography, ethic*, informed consent, privacy, confidential, education, guideline*, policy, teaching, and learning. The search strategy undertaken yielded 514 articles after the duplicates were removed (Figure 1). The inclusion criteria included:

- peer-reviewed reports of original research;

- literature published in the English language within the last 10 years; and

- exploration of the use of identifiable digital imagery for teaching and learning in the health care setting.

The 10-year range was chosen due to the advancements made in the area of digital photography, imaging, and smartphone technology in the past decade. ${ }^{18,19}$ Studies that examined histopathology specimens were excluded as these are unidentified specimens. Although integrative reviews allow the use of theoretical pieces, review articles, commentaries, editorials, gray literature, and narrative opinion, ${ }^{17}$ they were excluded. These abstracts were read by all authors (RK, VB,

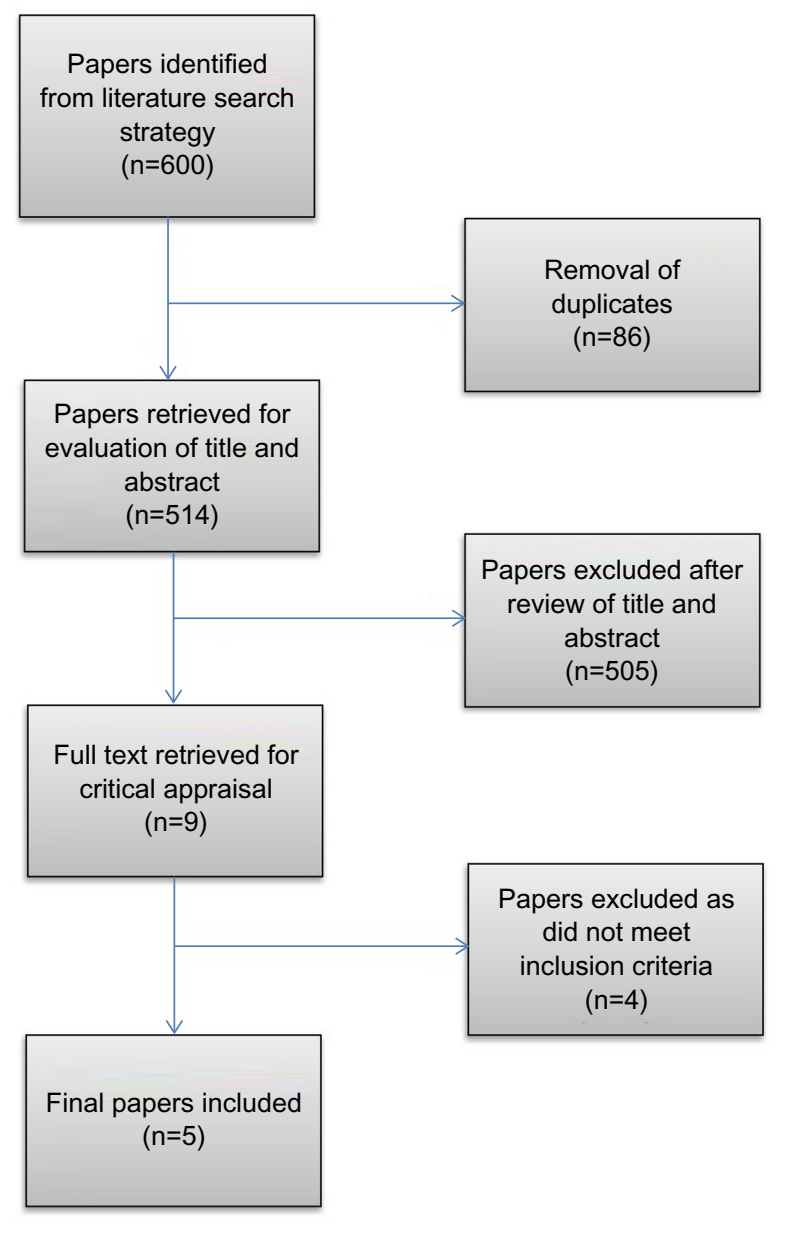

Figure I Flow diagram: literature review. 
and $\mathrm{RB}$ ) based on inclusion/exclusion criteria, yielding nine studies. Reading the full text of these studies resulted in the exclusion of four more articles as they did not meet the inclusion criteria, resulting in five studies deemed appropriate for inclusion in the review (Table 1). Any discrepancies experienced were resolved by active discussion until consensus was attained by all the parties. Findings were compiled and then arranged to identify themes and relationships.

\section{Results}

\section{Study characteristics}

This integrative review incorporated the key aspects of ethical implications of utilizing digital photography in the clinical setting for purposes of teaching and learning. Of the included studies, there were 678 participants collectively. The research was conducted within Australia and the UK. The respondents within the selected studies incorporate primarily nursing and medical professionals. The domains of the clinical practice settings included plastic surgery, dermatology, emergency medicine, and surgical/medical wards. All included studies were surveys/questionnaires with a response rate ranging from $22.6 \%$ to $78 \%$. The studies reported the use of digital photography/imaging in the clinical setting for the purpose of teaching and learning, including the issues of consent and utilization of technology. Surprisingly, out of the five studies, only one reported policies/guidelines in relation to digital photography in their results. ${ }^{20}$ The five studies were synthesized, and their findings categorized into three themes: knowledge deficit, consent and beyond, and standards driving scope of practice.

\section{Knowledge deficit}

Of significance was the notion of a deficit in knowledge pertaining to medical staff. It was clearly identified that having a poor understanding of the process of consent when capturing digital images and a lack of familiarity with the available policies had implications for ethical compliance and the potential for patient identification and harm.

Burns and Belton ${ }^{10}$ highlighted that ethical compliance may potentially be compromised when there is a deficit in comprehension and understanding. A knowledge deficit may lead to inappropriate and unsafe practice(s) in the clinical area, placing the patient at further risk of harm by compromising his/her privacy and identity. ${ }^{6}$ A knowledge deficit also renders health care professional at risk of breaching ethical standards and guidelines within their institutions, a finding reported by both Burns and Belton ${ }^{10}$ and Taylor et al. ${ }^{2}$ This demonstrates the need for educational workshops and further training within the clinical setting. An excerpt from an interview with a participant from the study of Burns and Belton ${ }^{10}$ clearly indicates a lack of awareness surrounding digital photography and the necessary process one must follow to comply with an ethical standard:

...everyone has phones that can take photos these days... but I was like 'ahh'... Where do you put that information?...

I do not fancy having it sitting around on a hard drive I have no control over.

Hubbard et $\mathrm{al}^{1}$ identified that dermatological trainees sought consent from consultants when taking digital images of patients, rather than obtaining consent from patients themselves. This raises questions and concerns around their understanding and perception of ethical process of digital photography. It is critical for clinicians to be aware of policies and guidelines concerning the process of digital photography, the ethical implications, and the potential for harm. To highlight this point, Hubbard et al, ${ }^{1}$ found that $33.6 \%$ of respondents were not aware of any available guidelines on the process of digital photography in their clinical area. Subsequently, despite a small number of facilities conducting an annual training program describing a protocol for obtaining, storing, and consenting digital images, Bhangoo et $\mathrm{al}^{20}$ found that these sessions were predominately attended by senior nursing staff with poor attendance by medical staff. This is surprising and concerning considering that most digital images of patients are taken by medical staff.

\section{Consent and beyond}

There remains an inadequacy of health care facilities to develop and implement policies and guidelines relevant to digital photography. Consequently, this has resulted in a disparity that currently exists between the taking of digital photographs for use in teaching and learning and the obtaining of informed patient consent for this specified purpose. However, agencies' guidelines such as the General Medical Council guidelines "Making and Using Visual and Audio Recordings of Patients in the United Kingdom"21 and acts such as the Health Insurance Portability and Accountability Act (HIPAA) ${ }^{6}$ provide clear guidance on when consent should be obtained and how it should be recorded and also address the taking of photographs for educational reasons, although this was not reflected in the studies included in this review.

Taylor et $\mathrm{al}^{2}$ identified that 25 of the 30 respondents in their study acquired digital images for the purpose of teaching and learning. Of the 25 respondents, ten always gained consent with two rarely and one never. The most common 


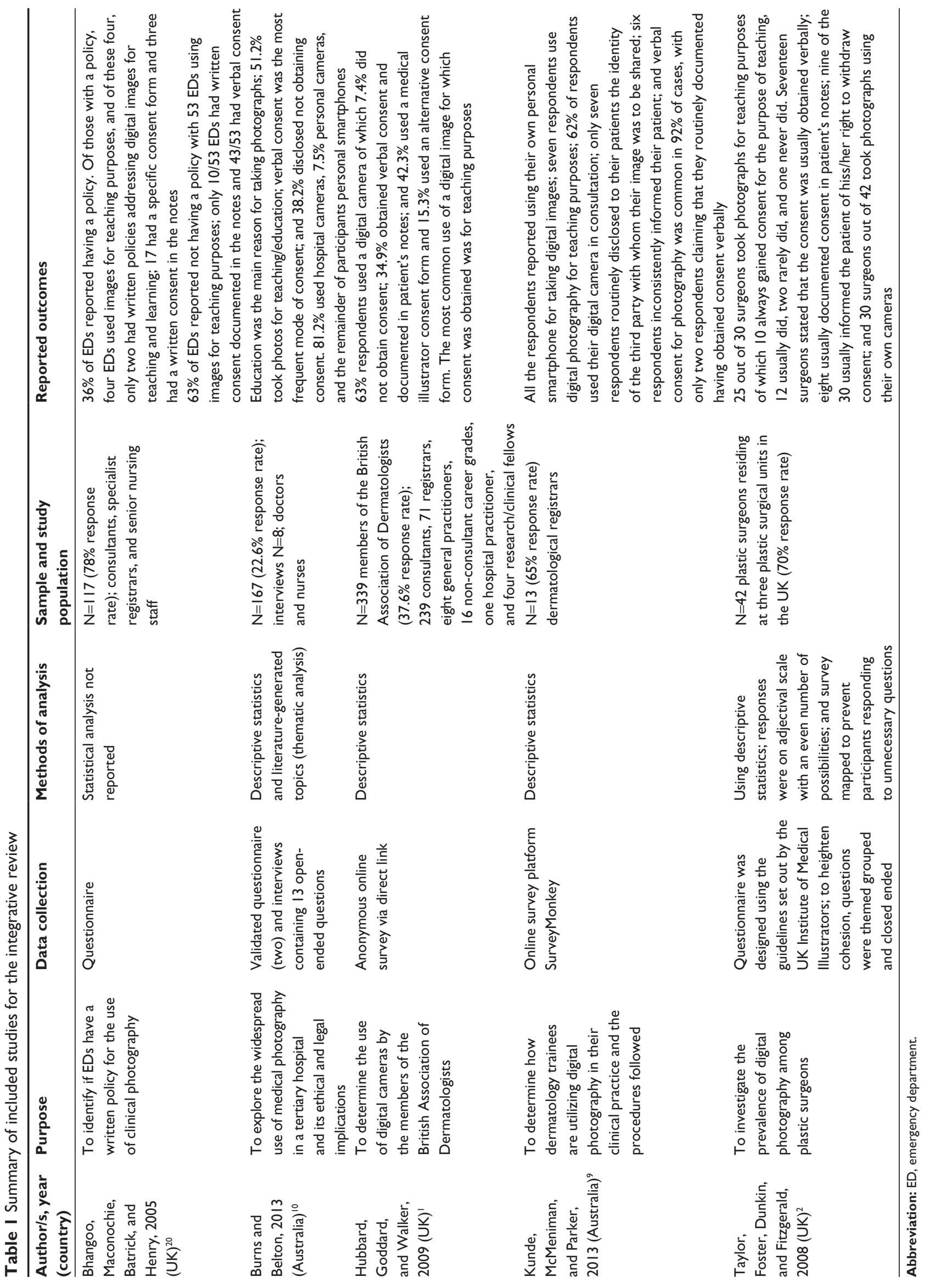


form of consent was verbal. Similarly, Burns and Belton ${ }^{10}$ found that 24 of 41 health care professionals reported verbal consent as the preferred method. This is problematic as there remains no data trail or evidence that the consent was sought or that the consent was obtained for the use of the images for purposes other than treatment, including teaching and learning. These concerns are echoed by a participant from Burns and Belton ${ }^{10}$ study stating:

I don't think people put significant enough emphasis on the consent process. I have often told people you know "make sure you have got consent" and they will go "oh yeah, yeah", but it's just for education.

This quote clearly emphasizes the lack of awareness concerning consent and the ethical implications of using the digital photography for educational purposes. Furthermore, the absence of consent was similarly identified by Hubbard et $\mathrm{al}^{1}$ who reported that $7.4 \%$ of respondents who used a digital camera to gain images for teaching and learning did so without consent. Kunde et $\mathrm{al}^{9}$ revealed that only $54 \%$ of their respondents reported that they regularly informed the patients of any third parties who may potentially view their image. While it may not always be possible for health care professionals to be specific about such viewing in a clinical setting, it remains important that we differentiate between the clinical and educational settings.

Patients routinely consent to the use of digital images for treatment purposes. However, the philosophy of obtaining written valid informed consent, prior to the acquisition of digital images for educational purpose, seems lacking. Taylor et $\mathrm{al}^{2}$ also emphasized the need for staff to inform patients that their consent may be withdrawn at any time, prior to images entering the public domain where they are irretrievable. However, withdrawing consent for the images regardless of the purpose of their acquisition may be a redundant notion given the speed of which data can be transferred and may well need to be addressed with the patient and their family.

\section{Standards driving scope of practice}

It is apparent from this review that within the clinical area, discrepancies exist around the availability of policies and guidelines related to digital photography. Of those policies that are available, the awareness of their existence by health care professionals appears to range from limited to 0 , posing concerns that they may not comply with these guidelines.

Given that the utilization of digital photography was for the purpose of teaching and learning across all the five included studies, it is essential that policymakers, health care practitioners, and administrators become familiar with ethical regulations and guidelines related to digital images. ${ }^{10}$ This is clearly highlighted in Bhangoo et al's ${ }^{20}$ findings that only $36 \%$ of emergency departments had a written policy pertaining to the taking of images, and of those, only two departments had a written policy specifically focused on photography in clinical and educational settings. To further highlight this issue, Taylor et $\mathrm{al}^{2}$ found that of the 25 surgeons in their study who took images for the purposes of teaching and learning, 17 took no extra measures to protect the anonymity of their patient.

Conversely, Hubbard et al ${ }^{1}$ did identify that in total 181 $(53.3 \%)$ respondents were aware of guidelines for digital photography, although $40(11.8 \%)$ stated that there were none and 114 (33.6\%) did not know whether there were any guidelines. This raises ethical concerns as there appears to be considerable variation in the specific guidance provided for the use of digital photography. It is also clear that health care professionals are partaking in the process of digital photography without recognizing the existence of policies and guidelines that guide safe practice, ensuring the patient's privacy is protected and no harm is perpetrated.

Despite Taylor et al's ${ }^{2}$ study emphasizing the importance of health professionals being aware of guidelines pertaining to digital photography, Burns and Belton ${ }^{10}$ study illustrates that noncompliance in the domain of obtaining consent for digital photography was widespread. Hence, it is evident that accessible, well-publicized policies and guidelines need to be developed to guide and safeguard those practicing digital photography within health care setting as well as the patients being photographed. ${ }^{20}$

\section{Discussion}

The authors have explored ethical considerations associated with the use of digital photography for teaching and learning purposes in the health care environment. Considering the fundamental importance that is directed toward ethics, informed consent, and patients' privacy, there are surprisingly few published studies exploring this domain.

\section{Implications for practice}

This review has identified several areas of concern. There is a lack of appropriate ethical guidelines to govern the use of digital photography for clinical education. We found that even when guidelines were available as demonstrated with the General Medical Council's "Making and Using Visual and Audio Recordings of Patients" 21 in the UK and the HIPAA in 
the US, ${ }^{6}$ health care personnel were either unaware of their existence or loath to follow them. To compound this, the definition of Protected Health Information under HIPAA, although only relevant to the US, is very broad and generalized and therefore does not specifically address the use of full facial images and images that could potentially reveal the identity of the patient. ${ }^{22}$ However, the studies reviewed revealed that when consent was obtained, it was commonly verbal, seemingly rarely informed, and often omitted reference to digital photographs being used for teaching and learning purposes. Finally, we found that those taking digital photographs had neither common understanding of how these photographs were to be stored securely nor with whom such images might be ethically shared.

While digital photography has become a simple, useful, and readily available tool to assist in diagnosis, it is becoming increasingly used by clinicians in areas including wound management as it provides an accurate and objective method of assessment and assists in with monitoring the progress of treatment. However, the use of such photographs for other purposes raises significant ethical questions, ${ }^{23}$ although we do not wish to obstruct the proper use of such images and strongly believe that the following steps should be undertaken to improve ethical practices and protect patients and clinicians:

1) Professional bodies should develop guidelines to assist in the acquisition and use of digital photographs for health education purposes.

2) Training programs should then be developed to raise awareness of these guidelines and local institutional policies to raise awareness and promote ethical conduct.

3) Appropriate consent is critical to the management of digital photographs. Consent should be informed, written, and obtained prior to any procedure and should include full disclosure of how images are to be taken, stored, and de-identified and how they are to be used and which audiences are likely to view them.

Appropriately acquired consent will protect both patients and staff from ethical dilemmas surrounding the use of digital photography within the domain of teaching and learning. In order to comply with ethical standards in the face of everchanging technology, the need for auditing of health care facilities on their practices around digital photography is critical and may shed light on the frequency and use of digital photography and the presence of policies and guidelines that reflect the current technological climate.

Emerging smartphone technology may provide some solutions in the management of digital images. Health care institutions globally have been incorporating the use of smartphone technology into their daily practice. ${ }^{6}$ For example, apps such as PicSafe ${ }^{\circledR}$ and Epic Haiku (Epic Systems Corporation, Verona, WI, USA) allow clinicians to consolidate the consent, capture and means to retain the image within a single program $^{10}$ and allows for integration into the medical record. Apps such as these may also be a critical tool for those who deliver education to clinicians and could be incorporated into existing policies and guidelines. However, it is essential that any policy or guideline must reflect and comply with the existing legislation. This has become increasingly difficult as technology is far outpacing legislative and legal systems. ${ }^{6}$ As such, this has resulted in some health care institutions deeming smartphone cameras as a significant ethical dilemma for patient privacy, resulting in the development and implementation of smartphone policies. ${ }^{6}$ Therefore, the development of policies and guidelines in accordance with the relevant legislation is warranted.

\section{Limitations and strength of evidence}

Strengths of this review include the use of three independent reviewers during the selection and extraction stages. No assumptions were made when methodology was unclear, which further strengthened the review process. Similarly, the development of logic tables and a search strategy across five major relevant databases, including the educational resources information database (Educational Resources Information Center), offer reassurance that the review is both rigorous and comprehensive.

This review is limited by the small number of original papers that were identified for evaluation. All papers included in this review were questionnaires, which may limit the strength of the findings. However, given the methodological complexities of evaluating issues as ethical implications, surveys can still offer useful and practical evidence that has the potential to guide policy and for guideline development. As the majority of studies in this review involved voluntary participation in questionnaires, there is potential for bias within the results. Likewise, small sample sizes and self-reporting may also impact data quality and the strength of our results. Finally, this review incorporates studies from Australia and the UK and as such may be perceived as limiting for it does not reflect a global perspective.

Despite the fact that the database search was extensive and inclusive, it was limited to only English language publications over the past 10 years and we did not review the gray literature, and therefore, may have overlooked some relevant studies. 


\section{Conclusion}

This integrative review suggests that there is currently a deficit in knowledge pertaining to ethical considerations of clinicians in obtaining, storing, utilizing, and distributing digital photography within the clinical and teaching environment. These results have implications for informing the practices of those who utilize digital photography routinely in their practice, in particular, newly graduated health professionals, with the opportunity to lay the foundations and embed their future career in sound ethical practices that ensure the safety of their patients and themselves in a dynamic highly technological and litigious health care environment.

\section{Disclosure}

All authors declare no support from any organization for the submitted work. VB was previously employed by The University of Tasmania and RK is currently employed by The University of Tasmania. The authors report no other conflicts of interest in this work.

\section{References}

1. Hubbard VG, Goddard DJ, Walker SL. An online survey of the use of digital cameras by members of the British Association of Dermatologists. Clin Exp Dermatol. 2009;34(4):492-494.

2. Taylor D, Foster E, Dunkin CS, Fitzgerald AM. A study of the personal use of digital photography within plastic surgery. J Plast Reconstr Aesthet Surg. 2008;61(1):37-40.

3. Berle I. Clinical photography and patient rights: the need for orthopraxy. J Med Ethics. 2008;34(2):89-92.

4. Mahar PD, Foley PA, Sheed-Finck A, Baker CS. Legal considerations of consent and privacy in the context of clinical photography in Australian medical practice. Med J Aust. 2013;198(1):48-49.

5. Palacios-Gonzalez $C$. The ethics of clinical photography and social media. Med Health Care Philos. 2015;18(1):63-70.

6. Harting MT, DeWees JM, Vela KM, Khirallah RT. Medical photography: current technology, evolving issues and legal perspectives. Int J Clin Pract. 2015;69(4):401-409.
7. Blain C, Mackay M. The digital revolution. Br J Perioper Nurs. 2004; 14(11):494-497, 499.

8. Lau CK, Schumacher HH, Irwin MS. Patients' perception of medical photography. J Plast Reconstr Aesthet Surg. 2010;63(6):e507-e511.

9. Kunde L, McMeniman E, Parker M. Clinical photography in dermatology: ethical and medico-legal considerations in the age of digital and smartphone technology. Australas J Dermatol. 2013;54(3): 192-197.

10. Burns K, Belton S. Clinicians and their cameras: policy, ethics and practice in an Australian tertiary hospital. Aust Health Rev. 2013;37(4): 437-441.

11. Berle I. The principles of ethical practice in professional clinical photography. J Vis Commun Med. 2004;27(1):11-13.

12. Payne K, Tahim A, Goodson A, Delaney M, Fan K. A review of current clinical photography guidelines in relation to smartphone publishing of medical images. J Vis Commun Med. 2012;35(4):188-192.

13. Berle I. The ethical context of clinical photography. J Vis Commun Med. 2002;25(3):106-109.

14. Johns MK. Informed consent for clinical photography. JVis Commun Med. 2002;25(2):59-63.

15. Hill K. Consent, confidentiality and record keeping for the recording and usage of medical images. J Vis Commun Med. 2006;29(2):76-79.

16. Tranberg H, Rous B, Rashbass J. Legal and ethical issues in the use of anonymous images in pathology teaching and research. Histopathology. 2003;42:104-109.

17. Whittemore R, Knafl K. The integrative review: updated methodology. J Adv Nurs. 2005;52(5):546-553.

18. Sandler J, Gutierrez RJ, Murray A. Clinical photographs: the gold standard, an update. Prog Orthod. 2012;13(3):296-303.

19. Van der Rijt R, Hoffman S. Ethical considerations of clinical photography in an area of emerging technology and smartphones. J Med Ethics. 2014;40(3):211-212.

20. Bhangoo P, Maconochie IK, Batrick N, Henry E. Clinicians taking pictures - a survey of current practice in emergency departments and proposed recommendations of best practice. Emerg Med J. 2005;22(11):761-765

21. General Medical Council. Making and Using Visual and Audio Recordings of Patients. General Medical Council; 2013. London.

22. Segal J, Sacopulos MJ. Photography consent and related legal issues Facial Plast Surg Clin North Am. 2010;18(2):237-244.

23. Crisp J, Taylor C, Douglas C, Rebeiro G. Potter and Perry's Fundamentals of Nursing - Australian Version - INKLING. Elsevier Health Sciences APAC; 2012.
Journal of Multidisciplinary Healthcare

\section{Publish your work in this journal}

The Journal of Multidisciplinary Healthcare is an international, peerreviewed open-access journal that aims to represent and publish research in healthcare areas delivered by practitioners of different disciplines. This includes studies and reviews conducted by multidisciplinary teams as well as research which evaluates the results or conduct of such teams or

\section{Dovepress}

healthcare processes in general. The journal covers a wide range of areas and welcomes submissions from practitioners at all levels, from all over the world. The manuscript management system is completely online and includes a very quick and fair peer-review system. Visit http://www.dovepress.com/testimonials.php to read real quotes from published authors. 